\title{
A new view on Auger data and cosmogenic neutrinos in light of different nuclear disintegration and air-shower models
}

\author{
Anatoli Fedynitch ${ }^{* * 1,2}$, Jonas Heinze ${ }^{2}$, Denise Boncioli ${ }^{2,3,4}$, and Walter Winter ${ }^{2}$ \\ 1) Dept. of Physics, University of Alberta, Edmonton, Alberta, Canada T6G 2E1 \\ 2) Deutsches Elektronen-Synchrotron (DESY), Platanenallee 6, D-15738 Zeuthen, Germany ${ }^{\dagger}$ \\ 3) Gran Sasso Science Institute (GSSI), Viale Francesco Crispi 7, 67100 L'Aquila, Italy \\ 4) INFN, Laboratori Nazionali del Gran Sasso (LNGS), 67100 Assergi, L'Aquila, Italy \\ ${ }^{\ddagger}$-mail: anatoli.fedynitcheualberta.ca
}

\begin{abstract}
We study the implications of Ultra-High Energy Cosmic Ray (UHECR) data from the Pierre Auger Observatory for potential accelerator candidates and cosmogenic neutrino fluxes for combinations of nuclear disintegration and air-shower models. We exploit the most recently published spectral and mass composition data (2017) with a new, computationally efficient simulation code PriNCe. We extend a systematic framework, which has been previously applied in a combined fit by the Pierre Auger Collaboration, with the cosmological source evolution as an additional free parameter. In this framework, an ensemble of generalized UHECR accelerators is characterized by a universal spectral index (equal for all injection species), a maximal rigidity, and the normalizations for five nuclear element groups. We find that the 2017 data favor a small but constrained contribution of heavy elements (iron) at the source. We demonstrate that the results moderately depend on the nuclear disintegration (PSB, Peanut, or Talys) model, and more strongly on the air-shower (EPOS-LHC, Sibyll-2.3, or QGSjet-II-04) model. Variations of these models result in different source evolutions and spectral indices, limiting the interpretation in terms of a particular class of cosmic accelerators. Better constrained parameters include the maximal rigidity and the mass composition at the source. Hence, the cosmogenic neutrino flux can be robustly predicted. Depending on the source evolution at high redshifts the flux is likely out of reach of future neutrino observatories in most cases, and a minimal cosmogenic neutrino flux cannot be claimed from data without assuming a cosmological distribution of the sources.
\end{abstract}

36th International Cosmic Ray Conference -ICRC2019-

July 24th - August 1st, 2019

Madison, WI, U.S.A.

\footnotetext{
* Speaker.

$\dagger$ This work has been supported by the European Research Council (ERC) under the European Union's Horizon 2020 research and innovation programme (Grant No. 646623)
} 


\section{Introduction}

The two largest detectors ever built, the Pierre Auger Observatory [1] and the Telescope Array [2], investigate the origin and the nature of Ultra-High Energy Cosmic Rays (UHECRs) above $10^{18}$ $\mathrm{eV}$ with hybrid detection techniques that combine signals from surface and fluorescence detectors to reconstruct extensive air showers. However, an association with a concrete source or class of sources is not yet in reach.

In this work, we revisit the approach of fitting the UHECR spectrum and composition using a density of homogeneously distributed "generic" UHECR sources as in the Auger's Combined fit (CF) [3]. In this high-energy approximation, the extragalactic transport is assumed to be purely ballistic, and hence diffusion due to the presence of magnetic fields is not taken into account. We study the impact of the model uncertainties on the astrophysical interpretation by performing scans in the three parameters: maximum rigidity $R_{\max }[\mathrm{GV}]$ (corresponding to the maximum energy of acceleration divided by the charge of the particle, $E_{\max } / Z$ ), spectral index $\gamma$ and cosmological evolution index $m$, using different combinations of nuclear disintegration and air-shower models. The computational requirements are significantly reduced through the new numerical code PRINCE, Propagation including Nuclear Cascade equations, that performs the propagation very efficiently under changing physical conditions. We investigate the three-dimensional source parameter space with a comparable resolution in all parameters for permutations of photo-nuclear disintegration and hadronic interaction models. Under the assumption of one dominant source population that accelerates cosmic ray nuclei up to a maximal rigidity, we compute the expected cosmogenic neutrino fluxes and discuss the robustness of the predictions by studying the major model uncertainties. This paper is an executive summary of the work published as [4].

\subsection{Simulation of extragalactic propagation}

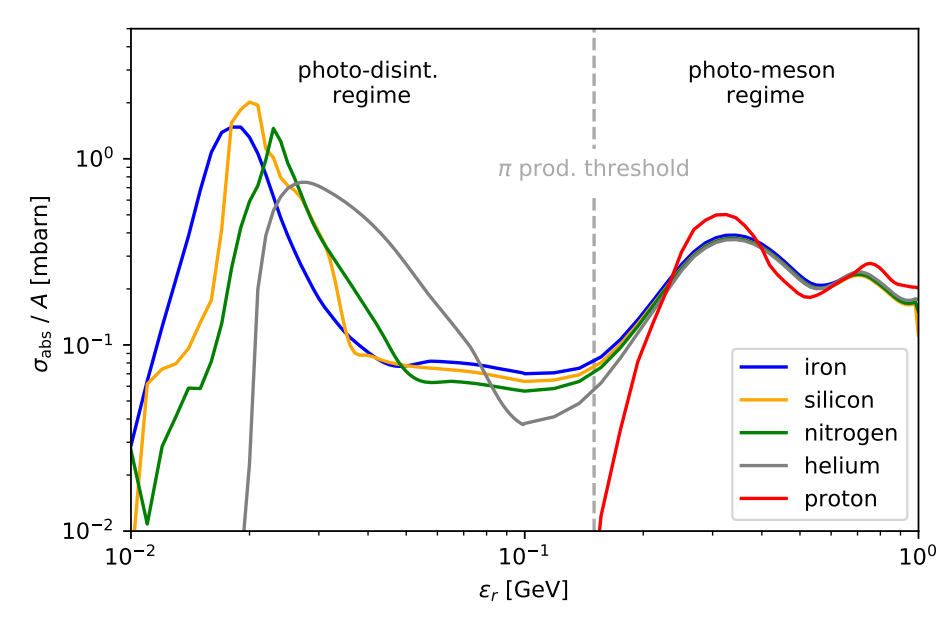

Figure 1: Photo-nuclear interaction cross section parameterization based on the PEANUT/FLUKA [5]. The dominant process in the disintegration regime at lower energies is the Giant Dipole Resonance (GDR). Pion production sets in above $150 \mathrm{MeV}$, where the cross section scales roughly with the mass number $A$.

During extragalactic propagation, UHECRs interact with the cosmic microwave and infrared background (CMB and CIB) via photo-pair $\left(\mathrm{e}^{+} \mathrm{e}^{-}\right)$production and photo-nuclear processes. Additionally, all relativistic particles lose energy adiabatically due to the expansion of the Universe. Photo-nuclear interactions can be subdivided into two regimes: photo-disintegration $\left(\varepsilon_{r}<150\right.$ 
$\mathrm{MeV}$ ) and photo-meson production (above the pion production threshold, $\varepsilon_{r}>150 \mathrm{MeV}$ ), where $\varepsilon_{r}$ is the photon energy in the nuclear rest frame, as illustrated in Fig. 1. In the photo-disintegration regime, the target photons interact with one or two nucleons and collectively excite the nucleus into a resonant state, which subsequently decays emitting (evaporating) nucleons, heavier fragments or keV-MeV photons.

We simulated the intergalactic transport of cosmic rays using a new original computer code called PRINCE. Instead of using a Monte-Carlo method, PRINCE numerically solves a system of coupled partial differential equations (PDEs) for the comoving density $Y_{i}\left(E_{i}, z\right)$ for each particle species $i$

$$
\begin{aligned}
\partial_{t} Y_{i}= & -\partial_{E}\left(b_{\mathrm{ad}} Y_{i}\right)-\partial_{E}\left(b_{e^{+} e^{-}} Y_{i}\right) \\
& -\Gamma_{i} Y_{i}+\sum_{j} Q_{j \rightarrow i}\left(Y_{j}\right)+J_{i} .
\end{aligned}
$$

for an arbitrary distribution $J_{i}\left(E, z, A_{i}\right)$ of isotropically emitting and homogeneously distributed cosmic ray sources detailed below. The terms (in order of occurrence) represent adiabatic cooling, pair production, photo-nuclear interactions (interaction and decays; reinjection) and injection from sources. This system of PDEs in $E$ and $z$ is solved using a $6^{\text {th }}$-order stencil operator for the $E$ derivatives and backward differentiation functions (BDF) for the redshift dependence. The code is very efficient, propagating a system with protons in less than a second, and a system including nuclei up to iron in tens of seconds starting from redshift one. A variation of the input spectra, the mass composition, the target fields and the interaction cross sections in between calculations has no impact on computing time (details can be found in the Appendix of [4]).

The modeling of cascading secondary nuclei during propagation requires inelastic interaction cross sections and inclusive production cross sections. Such cross sections can be obtained either empirically from data as in the Puget-Stecker-Bredekamp (PSB) [6] parameterization, or by tabulating the output of more realistic nuclear models. Here, we use TALYS [7], a comprehensive pre-equilibrium and Hauser-Feshbach theory based code, and PEANUT [5, 8] - an event generator of the FLUKA package (see Ref.[9] for a discussion of these models and their uncertainties).

Since cosmogenic neutrinos are only produced in the photo-meson regime, the differences between their production by free nucleons and nuclei are very pronounced for UHECR propagation. The photo-disintegration threshold prevents nuclei reaching energies $>A \cdot 10^{10} \mathrm{GeV}$ where photo-meson production sets in on CMB target photons. Instead, pions and cosmogenic neutrinos are produced by nuclei at energies below the cutoff $\sim 10^{9} \mathrm{GeV}$ on the less abundant CIB target photons. There are two consequences; the neutrino flux peaks at lower energies $\sim 10^{8} \mathrm{GeV}$ and is significantly lower compared to the protons-on-CMB case.

\section{Source model and fitting}

In the interest of comparability we parameterize the generic source population in the same way as in the CF [3] and [10]:

$$
J_{A}(E)=\mathscr{J}_{A} f_{\text {cut }}\left(E, Z_{A}, R_{\max }\right) n_{\mathrm{evol}}(z)\left(\frac{E}{10^{9} \mathrm{GeV}}\right)^{-\gamma},
$$


in which the nuclear species $A$ (here ${ }^{1} \mathrm{H},{ }^{4} \mathrm{He},{ }^{14} \mathrm{~N},{ }^{28} \mathrm{Si}$ and ${ }^{56} \mathrm{Fe}$ ) share a common spectral index $\gamma$ and a maximal rigidity $R_{\max }=E_{\max } / Z_{\mathrm{A}}$. The $\mathscr{J}_{A}$ are free normalization constants representing the number of particles ejected from the sources per unit of time, comoving volume and energy. The functional form of the cutoff is arbitrary and we adopt the definition of the CF:

$$
f_{\text {cut }}(E)= \begin{cases}1 & , E<Z_{\mathrm{A}} R_{\max } \\ \exp \left(1-\frac{E}{Z_{\mathrm{A}} R_{\max }}\right) & , E>Z_{\mathrm{A}} R_{\max } .\end{cases}
$$

In Eq. (2.1), the parameterization for the source evolution with redshift is given by the function:

$$
n_{\mathrm{evol}}(z)=(1+z)^{m}
$$

For variable $m$, the function approximates all known continuous source density functions within the UHECR horizon $z \lesssim 1$. However, an extrapolation to higher redshift is necessary for the computation of cosmogenic neutrinos.

The simulation is performed for five representative injection elements (listed above) that escape from the source. The source model comes with eight free parameters: $R_{\max }, \gamma, m$ and free normalizations $\mathscr{J}_{A}$ of the injection elements. In addition, we allow for a shift $\delta_{\mathrm{E}}$ in energy within the systematic uncertainty given by Auger $( \pm 14 \%)$ [11]. For the "discrete" parameters the simulation is repeated on a fine grid covering the ranges $9.7<\log _{10}\left(R_{\max }\right)<11.7,-1.5<\gamma<2.5$ and $-6<m<6$ for each injection element. The energy scale and the $\mathscr{J}_{A}$ continuously float in the minimization of

$$
\chi_{\mathscr{F}}^{2}=\sum_{i} \frac{\left(\mathscr{F}\left(E_{i}\right)-\mathscr{F}_{\text {model }}\left(E_{i}, \delta_{E}\right)\right)^{2}}{\sigma_{i}^{2}}
$$

for the sum of the three observables $\mathscr{F}$ : the combined spectrum, the $\left\langle X_{\max }\right\rangle$ and $\sigma\left(X_{\max }\right)$ above $E_{\min }=6 \cdot 10^{9} \mathrm{GeV}$.

\section{Fit to Auger's 2017 spectrum and composition}

We perform a fit to the 2017 update of the spectrum and $\left\langle X_{\max }\right\rangle$ measured by Auger [11, 12]. The parameter space is shown in Fig. 2 for the combination of TALYS as disintegration model and SIBYLL 2.3 as air-shower model (parameter values and uncertainties are given in Table II of Ref. [4]). The $\chi^{2} /$ dof is closer to one, compared to a value of two and three obtained in fits with fixed energy scale and source evolution to the 2017 and 2015 data, respectively. A value for $\gamma \approx 1$, corresponding to Fermi acceleration with diffusive escape is within the $95 \%$ contour in the $\gamma-R_{\max }$ plane. However, the preferred spectral indices result in flat or almost monochromatic spectra $\gamma<1$. The $R_{\max }-m$ plane exhibits a "low" rigidity cutoff for every choice of the source evolution within the $95 \% \mathrm{CL}$. This is required by the composition data, in particular the $\sigma\left(X_{\max }\right)$, that suggests a clear separation among the mass spectra. This result can be interpreted as a signature of the preference of the data for the scenario in which the spectrum at Earth cuts off because the sources reach a maximal rigidity, instead of the case where the cut off is due to photo-disintegration during the propagation. 

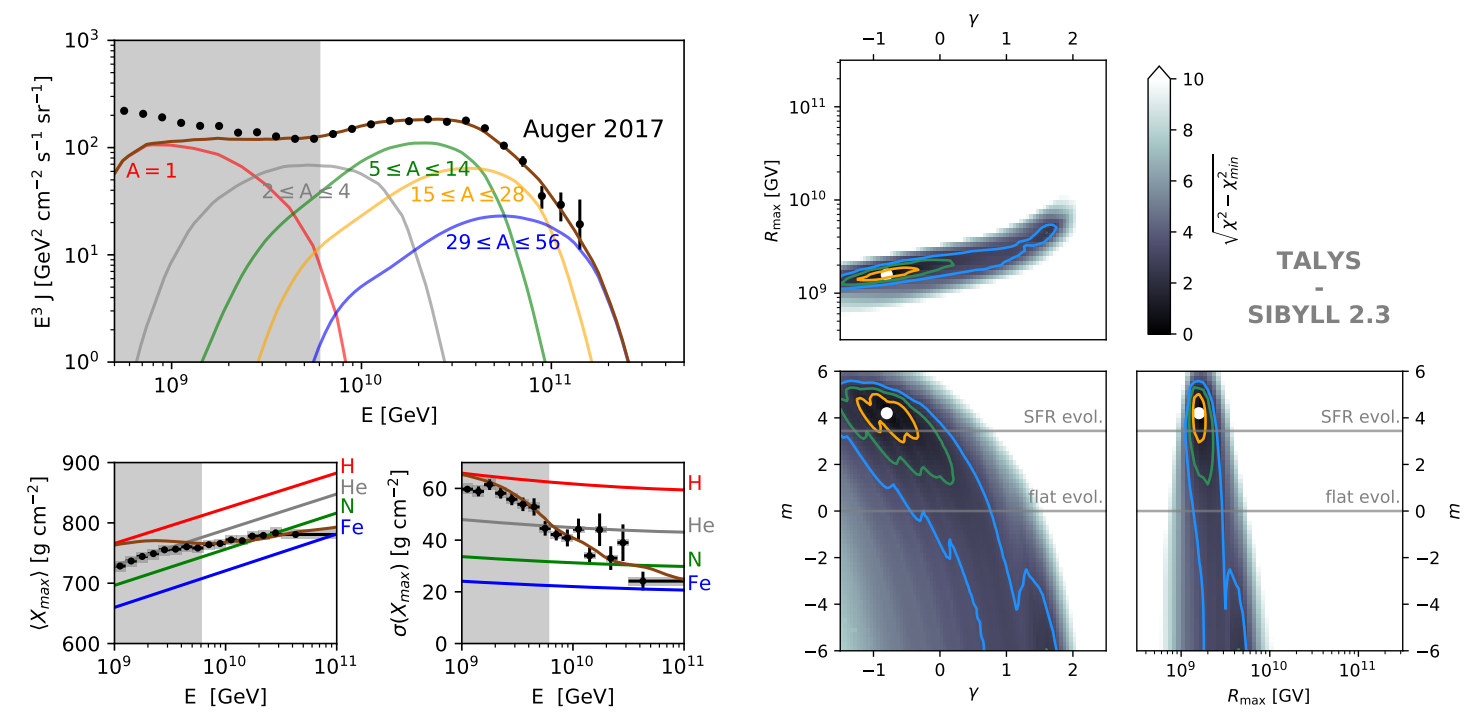

Figure 2: (Three subplots on the right) Parameter space in $\gamma, R_{\max }$ and $m$ for the model combination TALYS and Sib YLL 2.3. The best fit is found at $\gamma=-0.8, R_{\max }=1.6 \cdot 10^{9} \mathrm{GV}$ and $m=4.2$, and marked by a white dot. The colored shading corresponds to $\sqrt{\chi^{2}-\chi_{\min }^{2}}$, while $\chi^{2}-\chi_{\min }^{2}$ is used to determine the contours for $1 \sigma, 2 \sigma, 3 \sigma$ (for 2 d.o.f.). In each $2 \mathrm{D}$ panel, the third parameter is treated as a nuisance parameter and minimized over to project the 3D parameter space. (Subplots on the left) Spectrum (upper panel) and composition observables (lower panels) corresponding to the best fit to the Auger 2017 data [11, 12] for the combination TALYS and SiBYLL 2.3. Figures taken from [4].

The $\gamma-m$ parameter plane exhibits a clear anti-correlation, as already noticed for example in $[13,14]$. Positive source evolutions $(m>0)$ result in a pile up from more distant sources, effectively softening the spectrum at Earth. This pile up is compensated by harder spectra at the source. Contrariwise, a high density of local sources $(m<0)$ allows for spectral indices compatible with Fermi acceleration.

The spectrum and composition corresponding to the best fit of our baseline model are reported in the left panels of Fig.2. The pile-up effect from higher redshifts is clearly visible: While the injection spectrum is very hard $(\gamma=-0.8)$, the propagated spectra are softer and have a stronger overlap. The best fit for the proton component is 0 , and the proton component in the propagated spectrum comes only from propagation.

This result clearly favors positive evolutions, covering star-forming objects, GRBs and Blazars. The very hard spectra found in this case are consistent with what was found for example in [14]. The $3 \sigma$ contours leave room for negatively evolving sources such as TDEs [15].

\section{Model dependence of the UHECR fit}

The results for combinations of air-shower and disintegration model are shown in Fig. 3 for the projection to the $\gamma-m$ plane. We find satisfactory best fits with $\chi^{2} / \mathrm{dof} \approx 1.4-2.0$ for most model combinations, except for fits with QGSJETII-04 due to the model's broad $X_{\max }$ distributions in conjuncture with a small $\left\langle X_{\max }\right\rangle$. Clearly, the shower model has a stronger impact on the fit contours 

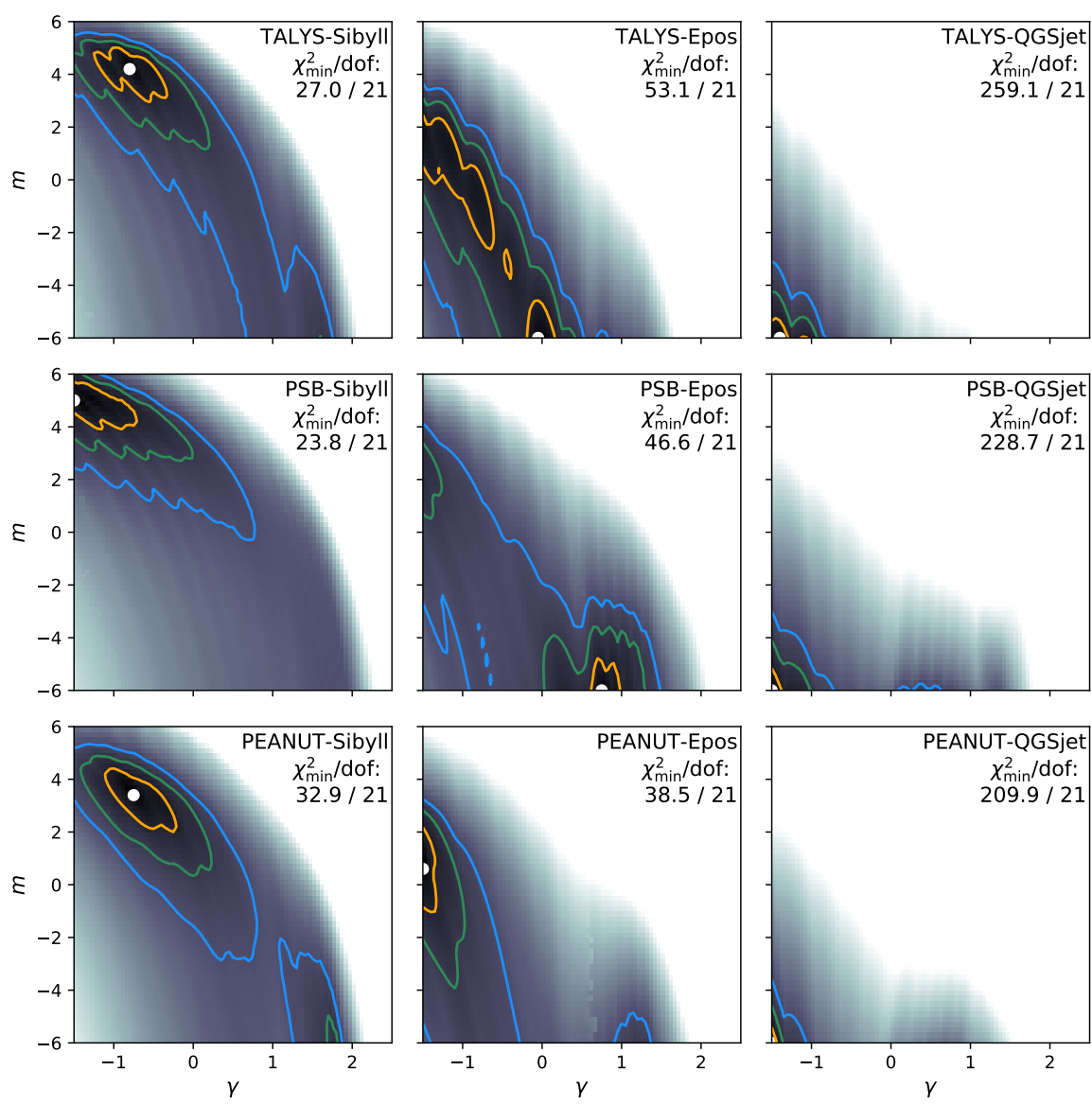

Figure 3: Parameter space in $\gamma$ and $m$ minimized over the third parameter $R_{\max }$ for different combinations of disintegration and air-shower models. The color code and contours are defined as in Fig. 2. Rows from top to bottom: Talys, PSB, Peanut. Columns from left to right: Sibyll 2.3, Epos-LHC, QGSJetiI-04. Figure taken from [4].

than the disintegration model, as can be seen comparing the columns in Fig. 3. The anti-correlation between $m$ and $\gamma$ persists among all model combinations (excluding QGSJETII-04).

Interestingly, when exchanging SiBYLL 2.3 with EpOS-LHC, the $3 \sigma$ contour in Fig. 3 is shifted towards more local sources and/or more monochromatic spectra. The $\chi_{\min }^{2} /$ dof is slightly worse for EPOS-LHC $(\approx 2.0)$ compared to SIBYLL $2.3(\approx 1.4)$, mainly because the fit to the $\left\langle X_{\max }\right\rangle$ is worse. It is however not strong enough to discriminate between these models, as the difference can be somewhat alleviated by allowing for shifts in $X_{\max }$ within the systematic uncertainties, which we did not include.

\section{Cosmogenic neutrinos}

Under the assumption that the fit is sensitive up to a redshift of $z_{\max }=1$, we draw in Fig. 4 the neutrino ranges corresponding to the 1,2 and $3 \sigma$ contours of the fit with the baseline model combination. Essentially, these flux levels can be regarded as constrained by present data. In contrast 

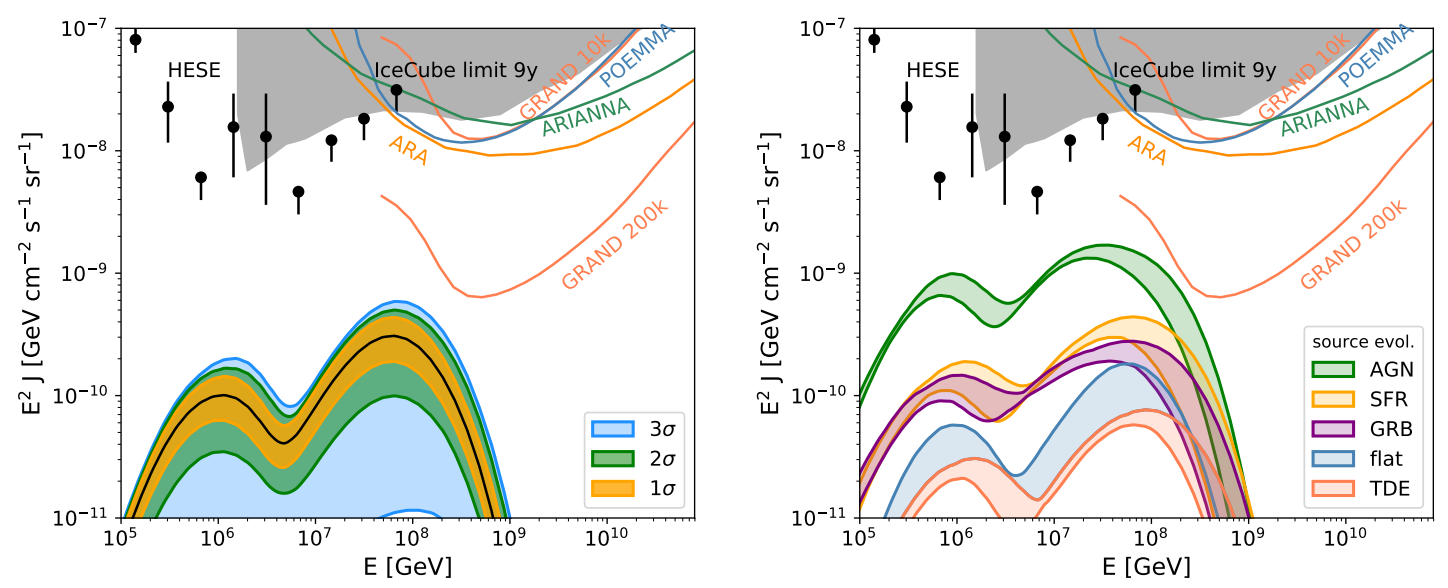

Figure 4: Left: Allowed range of the all-flavor neutrino flux from within the contours of the the 3D fit in Fig. 2. The source evolution is defined as $(1+z)^{m}$ for a maximum redshift $z_{\max }=1$. Estimated sensitivities for future radio neutrino detectors $[16,17,18,19]$. Right: All-flavor neutrino flux within the $3 \sigma$ region for the source evolution fixed to specific source classes and for a flat evolution. Figures taken from [4].

to the $1 \sigma$ region, which is limited to positive source evolutions, the $3 \sigma$ region is unconstrained towards negative redshifts (compare with Fig. 2). Hence, if the sources are local, the expected cosmogenic fluxes are very low.

The most significant impact on the fluxes comes from the extrapolation to redshifts $z>1$. In the right panel of Fig. 4, the flux is computed for fixed evolution functions of candidate source classes, where the parameter $m$ is not free; AGN [20, 21], GRB [22], SFR [23] (including starburst galaxies), TDE [24] and a flat evolution. In this case $z_{\max }=5$ is used, which is above the cutoff for all source evolutions used.

\section{Discussion}

While under the assumption of a dominant generic rigidity-dependent source type the data is sufficiently well described, a strong degeneracy in the parameter space remains. We have demonstrated that the reduced statistical error of the 2017 Auger data, in particular at the highest energy data points, favors for the first time a small but constrained iron fraction almost independent of the model variations. This implies a somewhat lower maximal rigidity. We find a clear indication of a correlation between the spectral index and source evolution: source candidates must be local $m<0$ with spectral indices compatible with those obtained in models with diffusive shock acceleration, or, distributed according to the star forming rate but with very hard, almost monochromatic, spectral indices.

We have assessed the impact of model variations on the contours in the $\gamma-m$ plane for all combinations of the disintegration models, finding the largest impact from air-shower modeling. The $3 \sigma$ contours enclose the entire range of $m$, implying that there is no clear preference for a candidate source type. For the associated cosmogenic neutrinos flux this means that no meaningful lower bound can be derived since local sources cannot be excluded by the fit. On the other hand, the upper bound is relatively robust under model variations. The fluxes are only constrained under 
fixed assumptions for the cosmic distribution of sources motivated by specific source classes. In all cases, the expected flux is small and peaks at energies around $10^{8} \mathrm{GeV}$ making the detection by the proposed future radio-based detectors unlikely. On the other hand, this result means that if very high energy neutrinos from sources exist at energies beyond $10^{8} \mathrm{GeV}$, the expected background from diffuse cosmogenic neutrinos is expected to be small. This conclusion applies if UHECRs are produced in one dominant type of accelerator with rigidity-dependent maximal energy cutoffs. If there are multiple types, for instance including a subset of proton rich sources, the fluxes can look significantly different.

In conclusion, our results demonstrate the limitations of what can be inferred from UHECR data alone and that the precision to which the UHECR mass composition is known plays a key role in obtaining constraints on source candidates and cosmogenic neutrinos.

\section{References}

[1] Pierre Auger Collaboration, A. Aab et al., Nucl. Instrum. Meth. A798 (2015) 172-213.

[2] Telescope Array Collaboration, T. Abu-Zayyad et al., Nucl. Instrum. Meth. A689 (2013) 87-97.

[3] Pierre Auger Collaboration, A. Aab et al., JCAP 1704 (2017) 038. [Erratum: JCAP1803,no.03,E02(2018)].

[4] J. Heinze, A. Fedynitch, D. Boncioli, and W. Winter, Astrophys. J. 873 (2019) 88.

[5] A. Ferrari, P. R. Sala, A. Fasso, and J. Ranft, CERN-2005-010 (2005).

[6] J. L. Puget, F. W. Stecker, and J. H. Bredekamp, Astrophys. J. 205 (1976) 638-654.

[7] A. J. Koning, S. Hilaire, and M. C. Duijvestijn, TALYS 1.0, in Proceedings, International Conference on Nuclear Data for Science and Tecnology, pp. 211-214, 2007.

[8] A. Fassò, A. Ferrari, and P. R. Sala, AIP Conference Proceedings 769 (2005) 1303-1306.

[9] D. Boncioli, A. Fedynitch, and W. Winter, Sci. Rep. 7 (2017) 4882.

[10] R. Alves Batista, R. M. de Almeida, B. Lago, and K. Kotera, arXiv:1806.10879.

[11] Pierre Auger Collaboration, F. Fenu, PoS ( ICRC2017) 486 (2018).

[12] Pierre Auger Collaboration, J. Bellido, PoS ( ICRC2017) 506 (2018).

[13] M. Unger, G. R. Farrar, and L. A. Anchordoqui, Phys. Rev. D92 (2015) 123001.

[14] A. M. Taylor, M. Ahlers, and D. Hooper, Phys. Rev. D92 (2015) 063011.

[15] D. Biehl, D. Boncioli, C. Lunardini, and W. Winter, Sci. Rep. 8 (2018) 10828.

[16] ARA Collaboration, P. Allison et al., Phys. Rev. D93 (2016) 082003.

[17] C. R. Persichilli. PhD thesis, University of California, Irvine, 2018.

[18] GRAND Collaboration, J. Alvarez-Muñiz et al., arXiv:1810.09994.

[19] J. Krizmanic, “Poemma: Probe of multi-messenger astrophysics.” UHECR, 2018.

[20] G. Hasinger, T. Miyaji, and M. Schmidt, Astron. Astrophys. 441 (2005) 417-434.

[21] T. Stanev, arXiv:0808.1045.

[22] D. Wanderman and T. Piran, "Mon. Not. Roy. Astron. Soc." 406 (Aug., 2010) 1944-1958.

[23] H. Yuksel, M. D. Kistler, J. F. Beacom, and A. M. Hopkins, Astrophys. J. 683 (2008) L5-L8.

[24] C. Lunardini and W. Winter, Phys. Rev. D95 (2017) 123001. 